\title{
Revivals, collapses and magnetic-pulse generation in quantum rings
}

\author{
A.S. Moskalenko* A. Matos-Abiague, and J. Berakdar \\ Max-Planck Institut für Mikrostrukturphysik, Weinberg 2, 06120 Halle, Germany
}

(Dated: May 10, 2019)

\begin{abstract}
Using a microscopic theory based on the density matrix formalism we investigate quantum revivals and collapses of the charge polarization and charge current dynamics in mesoscopic rings driven by short asymmetric electromagnetic pulses. The collapsed state is utilized for sub-picosecond switching of the current and associated magnetization, enabling thus the generation of pulsed magnetic fields with a tunable time structure and shape asymmetry which provides a new tool to study ultrafast spin-dynamics and ratchet-based effects.

PACS numbers: 73.23.-b,78.67.--n,72.15.Lh,42.65.Re
\end{abstract}

Introduction.- The spectacular advances in the design and tunability of the time-structure, amplitude, phase, and the shape of electromagnetic pulses [1, 2, 3, 4, 5, [6] have opened new avenues for their utilization in fundamental and applied research. Inducing and monitoring non-equilibrium states as they build up and decay [7, 8 ] are just but one example of recent applications. This progress in laser physics is paralleled with equally impressive development in the fabrication and manipulation of meso and nano-scale electronic systems in general, and of those with ring-confining geometry in particular 9, 10, 11, 12, 13, 14, 15. Subjecting quantum rings to strongly asymmetric pulses results in the formation of time-dependent charge polarization and charge currents 16, 17. How these non-equilibrium phases collapse, revive and eventually relax due to coupling to other degrees of freedom have not yet been addressed. We demonstrate that, once the evolution dynamics of the pulse-induced charge polarization is known, a scheme can be developed which allows to abruptly switch on and off the charge current in the ring, enabling thus a fine tuning of the time structure and the shape of the light-induced current. This is important in so far as the current in the ring is associated with a magnetic field, i.e. a controllability of the current renders the generation of short (down to picoseconds) magnetic pulses. The pulse-induced magnetic field can be utilized to study and manipulate locally and in a non-invasive way the picosecond spin-dynamics 18] of magnetic samples [19, 20, 21, 22]. In addition, we demonstrate how shape asymmetric magnetic pulses can be generated and controlled revealing thus the potential of our pulses for inducing a ratchet effect without spatially asymmetric potential 24]. The simplicity of the proposed setup and the local tunability of the magnetic pulse shape and duration make our scheme a valuable addition to currently known accelerator-based methods [19, 20, 21] and those based on the generation of currents in two photo switches excited by femtosecond laser pulses [23].

For inducing non-equilibrium states so-called electromagnetic half-cycle pulses (HCPs) [2, 3, 4] are employed. An HCP is a highly asymmetric monocycle pulse whose electric field consists of a strong and short (duration $\tau_{d} \gtrsim$ ps) half cycle followed by a much weaker but longer opposite polarity half cycle. Collapses and revivals of the HCPs-induced non-equilibrium charge polarization, the charge current generation and suppression, and relaxation effects are studied using the density-matrix formalism [8, 25]. As we are interested in non-destructive processes, weak HCPs (few $\mathrm{V} / \mathrm{cm}$ ) are applied. Thus, for low temperatures, $T$, the evolution dynamics is governed by coupling to longitudinal-acoustic (LA) phonons. Electron-electron interaction effects are suppressed by Pauli blocking and energy-conservation restrictions 26]. On the other hand, as we are interested in weak driving fields optical phonons do not influence the evolution dynamics because their energy is well above our highest excited electron energy.

Theory.- We consider $N$ electrons in an isolated ring with a mean radius $r_{0}$, height $z_{0}$, and width $d\left(z_{0} \ll d \ll\right.$ $r_{0}$ ) at low $T$. We specifically address the case where $d$ and $z_{0}$ are significantly smaller than the Fermi wave length of the charge carriers. Hence, only the lowest ground-state radial subbands are populated, a situation which is experimentally feasible for semiconductor-based rings 31]. The single-particle energies associated with the angular motion (characterized by the quantum number $m$ ) read $\varepsilon_{m}=\hbar^{2} m^{2} /\left(2 m^{*} r_{0}^{2}\right)$, where $m^{*}$ is the effective mass. The particles' angular dynamics is governed by the Hamiltonian $\hat{H}_{\text {tot }}=\hat{H}_{0}^{\text {carr }}+\hat{H}_{0}^{\text {phon }}+\hat{H}_{\mathrm{P}}+\hat{V}$. Here $\hat{H}_{0}^{\text {carr }}=$ $\sum_{m} \varepsilon_{m} a_{m}^{\dagger} a_{m}$ is the free carrier Hamiltonian and $a_{m}^{\dagger}\left(a_{m}\right)$ denote the creation (annihilation) operators. $\hat{H}_{0}^{\text {phon }}=$ $\sum_{\vec{q}} \hbar \omega_{\vec{q}}\left(b_{\vec{q}}^{\dagger} b_{\vec{q}}+\frac{1}{2}\right)$ is the free-phonon Hamiltonian, $\omega_{\vec{q}}$ is the frequency of a phonon with a momentum $\vec{q}$, and $b_{\vec{q}}^{\dagger}$ $\left(b_{\vec{q}}\right)$ are phonon creation (annihilation) operators. The carrier-phonon coupling is dictated by the Hamiltonian $\hat{H}_{P}=\sum_{\vec{q}, m, m^{\prime}} g_{\vec{q}}^{m^{\prime}} b_{\vec{q}} a_{m}^{\dagger} a_{m-m^{\prime}}+$ h.c., where $g_{\vec{q}}^{m-m^{\prime}}=$ $g_{\vec{q}}^{\text {bulk }} \int \mathrm{d}^{3} r \psi_{m}^{*}(\vec{r}) \mathrm{e}^{i \vec{q} \vec{r}} \psi_{m^{\prime}}(\vec{r})$ is the electron-phonon coupling constant 25] and $\psi_{m}(\vec{r})$ are wave functions of the ring carriers in the lowest radial subband. The bulk electron-LA-phonon coupling constant $g_{\vec{q}}^{\text {bulk }}$ is determined by $\left|g_{\vec{q}}^{\text {bulk }}\right|^{2}=\hbar|D|^{2} q /\left(2 V c_{\mathrm{LA}} \rho_{s}\right)$, where $D$ is the deformation constant, $c_{\mathrm{LA}}$ is the LA-velocity of sound, 
and $\rho_{s}$ is the lattice density. The interaction with a timedependent linearly polarized external electric field $F(t)$ is given by $\hat{V}=-e F(t) r_{0} \sum_{m, m^{\prime}}\left\langle m|\cos \phi| m^{\prime}\right\rangle a_{m}^{\dagger} a_{m^{\prime}}$. $\phi$ identifies the electron angular position with respect to the pulse polarization axis and $e$ is the electron charge.

A key aspect of this work is that the system is excited with a pulse which is very short on the relevant relaxation time scale. Hence, the excitation process can be separated from relaxation and dephasing which occur then in a field-free manner and can be monitored by measuring the emission spectrum. The excitation dynamics of the system is governed by Heisenberg's equation of motion (EOM) for the density operator $\hat{\rho}_{m, m^{\prime}}=a_{m}^{\dagger} a_{m^{\prime}}$, i.e., $i \hbar \partial_{t} \hat{\rho}_{m_{1}, m_{2}}=\left[\hat{H}_{0}^{\text {carr }}+\hat{V}, \hat{\rho}_{m_{1}, m_{2}}\right]$. In the interaction representation $\left(\hat{\rho}_{m_{1}, m_{2}}^{\text {int }}=\hat{\rho}_{m_{1}, m_{2}} \mathrm{e}^{\frac{i}{\hbar}\left(\varepsilon_{m_{2}}-\varepsilon_{m_{1}}\right) t}\right)$ we find

$$
\begin{aligned}
i \hbar \partial_{t} \hat{\rho}_{m_{1}, m_{2}}^{\text {int }}= & -\frac{1}{2} e F(t) r_{0}\left(\hat{\rho}_{m_{1}, m_{2}+1}^{\text {int }} \mathrm{e}^{\frac{i}{\hbar}\left(\varepsilon_{m_{2}}-\varepsilon_{m_{2}+1}\right) t}\right. \\
& +\hat{\rho}_{m_{1}, m_{2}-1} \mathrm{e}^{\frac{i}{\hbar}\left(\varepsilon_{m_{2}}-\varepsilon_{m_{2}-1}\right) t} \\
& -\hat{\rho}_{m_{1}+1, m_{2}} \mathrm{e}^{\frac{i}{\hbar}\left(\varepsilon_{m_{1}+1}-\varepsilon_{m_{1}}\right) t} \\
& \left.-\hat{\rho}_{m_{1}-1, m_{2}} \mathrm{e}^{\frac{i}{\hbar}\left(\varepsilon_{m_{1}-1}-\varepsilon_{m_{1}}\right) t}\right)
\end{aligned}
$$

If the applied pulse duration $\tau_{d}$ is much shorter than $\hbar /\left(\varepsilon_{m+1}-\varepsilon_{m}\right)$, where $\varepsilon_{m}$ is in the vicinity of the Fermi level $\left(E_{F}\right)$ within a range determined by $T$, then the exponential factors in Eq. (10) hardly vary on the time scale $\tau_{d}$. For low $T$ this situation is equivalent to $\tau_{d} \ll \tau_{F}$ where $\tau_{F}=2 \pi r_{0} / v_{F}$ and $v_{F}$ is the carrier Fermi velocity. This case (called impulsive approximation (IA) 27, 28]) is indeed realizable experimentally: for typical ballistic rings $\tau_{F}$ can be tens of picoseconds, while pulses down to subpicosecond time scale can be generated with contemporary techniques [2]. Under IA, Eq. (11) reduces in the basis-free form to $i \hbar \partial_{t} \hat{\rho}^{\text {int }}=\left[-e F(t) r_{0} \cos \phi, \hat{\rho}^{\text {int }}\right]$. If the duration of the negative HCP tail is much longer than $\tau_{F}$ the action of this tail on the system is averaged to zero. Applying the pulse at $t=0$ we operate again in the Schrödinger picture and derive for the density matrix the relation (for $t>\tau_{d}$ )

$$
\rho_{m_{1}, m_{2}}=\mathrm{e}^{\frac{i}{\hbar}\left(\varepsilon_{m_{1}}-\varepsilon_{m_{2}}\right) t} \sum_{m m^{\prime}} C_{m_{2}, m}^{*} C_{m_{1}, m^{\prime}} \rho_{m^{\prime}, m}^{0},
$$

where $\rho_{m^{\prime}, m}^{0}$ is the density matrix before the application of the pulse. $\quad C_{m_{1}, m} \equiv\left\langle m\left|\mathrm{e}^{i \alpha \cos \phi}\right| m_{1}\right\rangle=$ $i^{m_{1}-m} J_{m_{1}-m}(\alpha), J_{l}(x)$ are Bessel functions, and $\alpha=$ $r_{0} p / \hbar$, where $p=-e \int_{0}^{\tau_{d}} F(t) d t$ is the momentum transferred to the charge carriers. If for $t<0$ the system is in an equilibrium state with an initial density ma$\operatorname{trix} \rho_{m^{\prime}, m}^{0}=f_{m}^{0}(\eta, T) \delta_{m^{\prime}, m}$, where $f_{m}^{0}(\eta, T)$ is the FermiDirac distribution function ( $\eta$ and $T$ denote the chemical potential and the temperature, respectively) 32. For our isolated rings the chemical potential at a given temperature is uniquely determined by the number of particles in the ring $N$. The pulse electric field is chosen as $F(t)=F_{0} \frac{t}{\tau_{0}}\left[\exp \left(-\frac{t^{2}}{2 \tau_{0}^{2}}\right)-\frac{1}{b^{2}} \exp \left(-\frac{t}{b \tau_{0}}\right)\right]$. The parameters $F_{0}, \tau_{0}$, and $b$ determine respectively the amplitude, the duration, and the asymmetry of the pulse. The duration $\tau_{d}$ of the positive half cycle is determined by $\tau_{d}=\tau_{0}\left(1+\sqrt{1+2 b^{2} \ln b^{2}}\right) / b$. For $b=8$ the pulse has basically the experimentally observed shape and ratio of 13:1 between the maximum field values of the positive and negative polarity parts [2]. The peak field strength is $F_{\mathrm{p}} \approx 0.593 F_{0}$. When rings are irradiated with these pulses, a charge polarization builds up which is characterized by the dipole moment $\vec{\mu}(t)=\operatorname{Tr}[e \vec{r} \hat{\rho}(t)]$. The components of the dipole moment along and perpendicular to the pulse polarization are $\mu_{\|}(t)=e r_{0} \sum_{m} \operatorname{Re}\left[\rho_{m+1, m}\right]$ and $\mu_{\perp}(t)=e r_{0} \sum_{m} \operatorname{Im}\left[\rho_{m+1, m}\right]$, respectively. A detailed comparison between the dynamics of the dipole moment calculated using the IA and the exact numerical solution for different $\tau_{d}$ but fixed $\alpha$ endorsed our expectation that IA is well justified if $\tau_{d}$ is smaller than a quarter period of the dipole moment oscillations (a period of oscillation is determined by the energy difference between levels near $E_{F}$ ) and the HCP tail duration is longer than a quarter period of oscillations. Thanks to the IA a single parameter, the kick strength $\alpha$, is sufficient for characterizing the coupling of the HCP to the electronic system.

The non-equilibrium dynamics of the induced dipole moment is inferred from EOM for $\rho_{m_{1}, m_{2}}$ (including scattering from phonons), i.e., [25]

$$
\begin{aligned}
i \hbar \partial_{t} \rho_{m_{1}, m_{2}}= & \left(\varepsilon_{m_{2}}-\varepsilon_{m_{1}}\right) \rho_{m_{1}, m_{2}} \\
+ & \sum_{m_{3} \vec{q}}\left[g_{\vec{q}}^{m_{2}-m_{3}} s_{\vec{q}}^{m_{1}, m_{3}}+\left(g_{\vec{q}}^{m_{3}-m_{2}}\right)^{*}\left(s_{\vec{q}}^{m_{3}, m_{1}}\right)^{*}\right. \\
& \left.\quad-g_{\vec{q}}^{m_{3}-m_{1}} s_{\vec{q}}^{m_{3}, m_{2}}-\left(g_{\vec{q}}^{m_{1}-m_{3}}\right)^{*}\left(s_{\vec{q}}^{m_{2}, m_{3}}\right)^{*}\right] .
\end{aligned}
$$

Using the Markov approximation and neglecting polaron corrections the phonon-assisted density matrices $s_{\vec{q}}^{m_{1}, m_{2}} \equiv\left\langle b_{q} a_{m_{1}}^{\dagger} a_{m_{2}}\right\rangle-\left\langle b_{q}\right\rangle\left\langle a_{m_{1}}^{\dagger} a_{m_{2}}\right\rangle$ in absence of an external mechanical action read [25]

$$
\begin{aligned}
s_{\vec{q}}^{m_{1}, m_{2}}= & -i \pi \delta\left(\varepsilon_{m_{2}}-\varepsilon_{m_{1}}+\hbar \omega_{\vec{q}}\right) \sum_{m_{3}, m_{4}}\left(g_{\vec{q}}^{m_{4}-m_{3}}\right)^{*} \\
& \times\left[\left(n_{\vec{q}}+1\right) \rho_{m_{1}, m_{4}} \bar{\rho}_{m_{3}, m_{2}}-n_{\vec{q}} \rho_{m_{3}, m_{2}} \bar{\rho}_{m_{1}, m_{4}}\right],
\end{aligned}
$$

where $\bar{\rho}_{m, m^{\prime}} \equiv \delta_{m, m^{\prime}}-\rho_{m, m^{\prime}}$. For $\alpha<1$, i.e. for weak excitations, we write $\rho_{m_{1}, m_{2}} \approx f_{m_{1}}^{0} \delta_{m_{1}, m_{2}}+\tilde{\rho}_{m_{1}, m_{2}}$. Assuming thermal equilibrium for phonons [i.e., $n_{\vec{q}} \equiv n^{0}(q)$, where $n^{0}(q)$ is the Bose-Einstein distribution function], performing the sums over $\vec{q}$, and assuming the Debye model for the LA-phonon spectrum, we arrive at the equation for our numerical calculations of the relaxation dynamics

$$
\begin{aligned}
\partial_{t} \tilde{\rho}_{m_{1}, m_{2}}= & {\left[\frac{i}{\hbar}\left(\varepsilon_{m_{1}}-\varepsilon_{m_{2}}\right)-\sum_{m} \frac{R_{m_{1}}^{m}+R_{m_{2}}^{m}}{\tau_{\mathrm{LA}}}\right] \tilde{\rho}_{m_{1}, m_{2}} } \\
& +\sum_{m} \frac{\tilde{\rho}_{m_{1}+m, m_{2}+m}}{\tau_{\mathrm{LA}}}\left(R_{m_{2}+m}^{m_{1}}+R_{m_{1}+m}^{m_{2}}\right),
\end{aligned}
$$



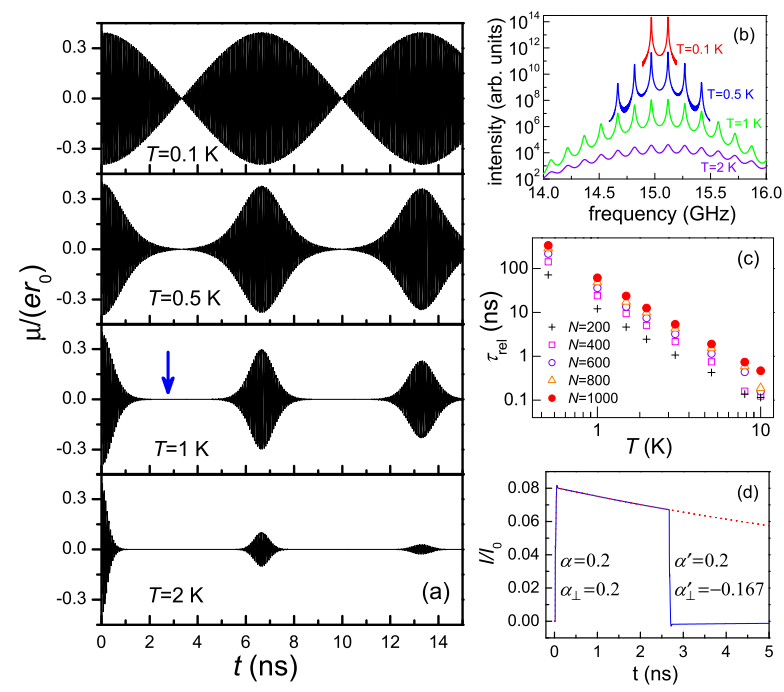

FIG. 1: (a) Dynamics of the dipole moment $\mu$ for different temperatures. Kick strength $\alpha=0.2$. (b) Emission spectra. The curves are offset vertically for clarity. (c) Dependence of the relaxation time of the dipole moment on temperature $T$ and the number $N$ of electrons. (d) Numerically calculated current decay (dotted line) after excitation by the $\frac{1}{4} T_{\mathrm{cl}^{-}}$ delayed sequence of two mutually perpendicular HCPs and current suppression (full line) by application of the second $\frac{1}{4} T_{\mathrm{cl}}$-delayed sequence. Duration of each HCP is $\tau_{d}=3 \mathrm{ps}$; $\mathrm{T}=1 \mathrm{~K}$. The arrow in (a) marks the time moment when the stopping sequence of HCPs is applied. Parameters of the ring: $r_{0}=1.35 \mu \mathrm{m}, d=50 \mathrm{~nm}$, and $N=400$.

where $\tau_{\mathrm{LA}}=\hbar c_{\mathrm{LA}}^{2} \rho_{s} d^{2} r_{0} /|D|^{2}$,

$$
\begin{aligned}
& R_{m^{\prime}}^{m}=\mathcal{F}_{m}^{m^{\prime}}\left(q_{m^{\prime}}^{m} d\right)\left[n^{0}\left(q_{m^{\prime}}^{m}\right)+f_{m}^{0}\right], \quad q_{m^{\prime}}^{m} \in\left(0, \frac{\omega_{\mathrm{D}}}{c_{\mathrm{LA}}}\right) \\
& R_{m^{\prime}}^{m}=\mathcal{F}_{m}^{m^{\prime}}\left(q_{m^{\prime}}^{m} d\right)\left[n^{0}\left(q_{m^{\prime}}^{m}\right)+1-f_{m}^{0}\right], \quad q_{m^{\prime}}^{m} \in\left(-\frac{\omega_{\mathrm{D}}}{c_{\mathrm{LA}}}, 0\right)
\end{aligned}
$$

$$
R_{m^{\prime}}^{m}=0
$$

otherwise;

$q_{m^{\prime}}^{m}=\left(\varepsilon_{m}-\varepsilon_{m^{\prime}}\right) /\left(\hbar c_{\mathrm{LA}}\right)$ and $\omega_{\mathrm{D}}$ is the Debye frequency; $\mathcal{F}_{m}^{m^{\prime}}(y)=8 \pi^{2} y \int_{0}^{y} \mathrm{~d} x \frac{1}{\sqrt{1-x^{2} / y^{2}}} \frac{\sin ^{2}(x / 2)}{x^{2}\left[x^{2}-(2 \pi)^{2}\right]^{2}}$ if $\operatorname{sgn}(m)=$ $\operatorname{sgn}\left(m^{\prime}\right)$ and $\mathcal{F}_{m}^{m^{\prime}}(y)=0$ otherwise.

Numerical results.- The short-pulse induced dynamics occurs within the following scenario. Starting from the ground state a short pulse drives the system to the excited state determined by Eq. (2). This non-equilibrium state serves as the initial condition for the numerical propagation of equation (3]), which in turn govern the post-pulse charge dynamics.

For the calculations the following ring material parameters (corresponding to $\mathrm{n}-\mathrm{GaAs}$ ) are employed: $c_{\mathrm{LA}}=$ $4.79 \times 10^{5} \mathrm{~cm} / \mathrm{s}, \rho_{s}=5.32 \mathrm{~g} / \mathrm{cm}^{-3},|D|=8.6 \mathrm{eV}, \hbar \omega_{\mathrm{D}}=$ $30 \mathrm{meV}, m^{*}=0.067 m_{0}$ ( $m_{0}$ is the free electron mass). Fig. [1 (a) shows the nanosecond-dynamics of the dipole moment $\mu$ for $N=400$ and different $T$ (the oscillatory behavior on the much shorter time scale $\tau_{F}$ is not resolved [16]). For low $T(=0.1 \mathrm{~K})$ only states with two possible energy values near the $E_{F}$ are populated. The long-time behavior exhibits thus beatings and hence two peaks in the emission spectrum (Fig. 10(b)) are observable. With increasing $T$ more levels near $E_{F}$ are populated and the dynamics of the dipole moment shows alternating collapsed states and quantum revivals. The revival time is given by $T_{\mathrm{rev}}=4 \pi \hbar /\left|\partial^{2} \varepsilon_{m} / \partial m^{2}\right|$ with $\varepsilon_{m}$ in the proximity of $E_{F}$. The fast (classical) oscillations have a period $T_{\mathrm{cl}}=2 \pi \hbar /\left|\partial \varepsilon_{m} / \partial m\right| \approx \tau_{F}[29]$. The decay of the revival peak values is due to the relaxation. For an estimate of the dipole-moment relaxation time, $\tau_{\text {rel }}$, we utilize the decay dynamics and use the two first consecutive envelope maxima to determine $\tau_{\text {rel }}$ from a fit by $A \exp \left(-t / \tau_{\text {rel }}\right)$. Fig. 1(c) displays the dependence of $\tau_{\text {rel }}$ on $T$ and on the number of electrons $N$ in the ring. $\tau_{\text {rel }}$ increases rapidly with decreasing $T$ and, as expected, $\tau_{\text {rel }}$ tends to infinity for $T \rightarrow 0$. Fig. 目 (b) evidences that by measuring the emission spectrum the temperature-dependent decay dynamics can be traced. The different decay dynamics of the dipole moment and of the current (which can be monitored separately) allows insights into different parts of the density matrix. The dipole moment (current) is namely determined by the diagonal (near-diagonal) elements of the density matrix.

Magnetic field tuning.- Applying two time-delayed mutually perpendicular HCPs generates charge current $I$ and an associated magnetization $M$ in the ring 17]. The utilization of the pulse-induced magnetic field to monitor locally, in a pump-probe manner, the ultra-fast spin dynamics in nanostructures or to induce a fast ratchet effect require a fine control of the fast switching behavior of the pulse-triggered magnetic field, a task which is tackled here for the first time by utilizing the collapsed states of the dipole moments. Two weak perpendicular pulses with strengths $\alpha_{\|}$and $\alpha_{\perp}$ and delay time $\tau$ (chosen as $\tau=T_{\mathrm{cl}} / 4$ ) initiate along the respective field axis two independent polarization dynamics $\mu_{\|}(t)$ and $\mu_{\perp}(t)$. Using Eq. (2) we derive that the charge current change $\Delta I$ by application of the second HCP with kick strength $\alpha_{\perp}$ at $t=t_{\mathrm{sw}}$ is given by

$$
\Delta I=-\alpha_{\perp} \frac{\mu_{\|}\left(t=t_{\mathrm{sw}}^{-}\right)}{e r_{0}} I_{0}, \quad I_{0}=e \hbar /\left(m^{*} r_{0}^{2}\right)
$$

Correspondingly, the ring magnetization is shifted by $\Delta M=\pi r_{0}^{2} \Delta I$, and the current-generated magnetic flux by $\Delta \Phi=\frac{\pi}{2} \mu_{0} r_{0} \Delta I$ ( $\mu_{0}$ is the magnetic permeability of surrounding material). Eq. (5) can be exploited to turn the current on and off producing thus short magnetic pulses, as follows: Having produced a current with a HCPs pair we subject (after the time $\tau_{p-p}$ ) the collapsed state to a second sequence of two pulses. The first pulse of the second sequence has the strength $\alpha^{\prime}=\alpha_{\|}$ and triggers the same dynamics of the dipole moment as does the first HCP of the first HCPs pair because the collapsed state is unpolarized. The second pulse of the second sequence has strength $\alpha_{\perp}^{\prime}$ and is applied with 

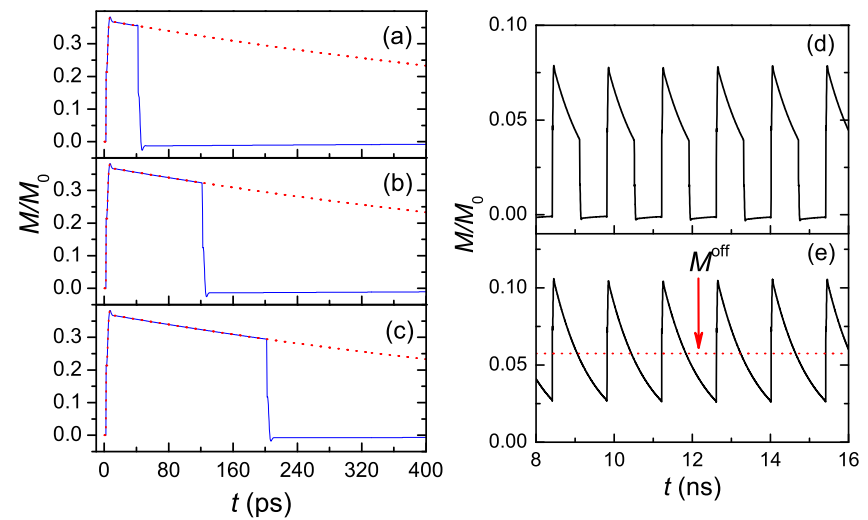

FIG. 2: Magnetization pulses generated in the ring by application of two HCP pairs delayed by $\tau_{\mathrm{p}-\mathrm{p}}=40 \mathrm{ps}$ in (a), $\tau_{\mathrm{p}-\mathrm{p}}=120 \mathrm{ps}$ in (b), and $\tau_{\mathrm{p}-\mathrm{p}}=200 \mathrm{ps}$ in (c). Kick strengths are $\alpha_{\|}=\alpha_{\perp}=\alpha_{\|}^{\prime}=0.4, \alpha_{\perp}^{\prime}=-0.39$ in (a), $\alpha_{\perp}^{\prime}=-0.35$ in (b), and $\alpha_{\perp}^{\prime}=-0.32$ in (c). Magnetization $M$ is normalized to $M_{0}=\pi r_{0}^{2} I_{0}$. Dotted lines show the result without applying the second HCP pair. Parameters of the calculation: $r_{0}=0.3 \mu \mathrm{m}, d=20 \mathrm{~nm}, \mathrm{~N}=160, \mathrm{~T}=20 \mathrm{~K}$, $\tau_{d}=0.5$ ps. Figures (d) and (e) show periodically alternating time-asymmetric magnetization generated in the ring by application of series of sequences of HCPs with a time delay between sequences $1.4 \mathrm{~ns}$. In (d) each sequence contains four HCPs having kick strengths $\alpha_{\|}=0.2, \alpha_{\perp}=0.2, \alpha_{\|}^{\prime}=0.2$, and $\alpha_{\perp}^{\prime}=-0.1$, respectively. In (e) each sequence contains two HCPs having kick strengths $\alpha_{\|}=0.2$ and $\alpha_{\perp}=0.2$, respectively. $M^{\text {off }}$ is the offset magnetization associated with an external magnetic field 24]. Parameters of the ring and duration of HCPs as in Fig. 1 $T=4 \mathrm{~K}$.

polarity opposite to the second HCP of the first pair. $\left|\alpha_{\perp}^{\prime}\right|$ is chosen smaller than $\alpha_{\perp}$ by a factor determined by the current decay during $\tau_{\mathrm{p}-\mathrm{p}}$ and in such a way that the currents triggered by the two HCP pairs cancel each other. Fig. 1(c) demonstrates numerically the accuracy of this scheme of current switching. The abrupt current switching opens the way for the tunability of the timestructure of the magnetic pulses. The magnetic pulses duration is stretchable depending on $\tau_{\mathrm{p}-\mathrm{p}}$ (cf. Fig. 2/a)(c)): As inferred from Figs. 1(d) 2(a)-(c), if $\tau_{\mathrm{p}-\mathrm{p}} \ll \tau_{\text {rel }}^{\text {cur }}$, where $\tau_{\text {rel }}^{\text {cur }}$ is the time constant for the current initial decay dynamics, almost rectangular ps magnetic pulses are produced. For $\tau_{\mathrm{p}-\mathrm{p}} \gtrsim \tau_{\text {rel }}^{\text {cur }}$ asymmetric pulses are triggered, which is of importance when studying ratchet effects [24]. Fig. 2] demonstrates the level of tunability of the pulse shape and duration by varying experimentally accessible parameters. The magnitude of the triggered pulses is enlarged for smaller rings and/or stronger kicks. Also an appropriate arrangement of a collection of rings allows additional tuning of the magnetic pulses.

In summary, we demonstrated how relaxation, collapses, and revivals in quantum rings can be studied by means of electromagnetic pulses and how these phenomena can be exploited to generate with current technology magnetic pulses with tunable time and shape structure.
Numerical calculations with realistic pulse and material parameters are performed endorsing the feasibility of the predicted effects with nowadays technology.

* Electronic address: moskalen@mpi-halle.de Also at A.F. Ioffe Physico-Technical Institute, 194021 St. Petersburg, Russia

[1] A.M. Weiner, D.E. Laird, J.S. Patel, J.R. Wullert, Opt. Lett. 15, 326 (1990); M.M. Wefers, K.A. Nelson, ibid 18, 2032 (1993).

[2] D. You, R. R. Jones, P. H. Bucksbaum, D. R. Dykaar, Opt. Lett. 18, 290 (1993); N. E. Tielking, T. J. Bensky, R. R. Jones, Phys. Rev. A 51, 3370 (1995).

[3] T. J. Bensky, G. Haeffler, and R. R. Jones, Phys. Rev. Lett. 79, 2018 (1997).

[4] G. L. Carr et al., Nature 420, 153 (2002); M. Sherwin, Nature 420, 131 (2002).

[5] T. Brabec and F. Krausz, Rev. Mod. Phys. 72, 545 (2000); R. E. Slusher, ibid 71, 471 (1999).

[6] T. Brixner et al., Phys. Rev. Lett. 92, 208301 (2004).

[7] R. Huber et al., Nature 414, 286 (2001).

[8] H. Haug, S. W. Koch, Quantum Theory of the Optical and Electronic Properties of Semiconductors (World Scientific Publishing, London, 2004).

[9] D. Mailly, C. Chapelier, A. Benoit, Phys. Rev. Lett. 70, 2020 (1993).

[10] A. Fuhrer et al., Nature 413, 822 (2001).

[11] A. Lorke et al., Phys. Rev. Lett. 84, 2223 (2000).

[12] W. Rabaud et al., Phys. Rev. Lett. 86, 3124 (2001).

[13] Mohanty, Ann. Phys. 8, 549 (1999).

[14] E.M.Q. Jariwala, P. Mohanty, M.B. Ketchen, and R.A. Webb, Phys. Rev. Lett. 86, 1594 (2001).

[15] M. Kläui et al., Appl. Phys. Lett. 85, 5637 (2004); Phys. Rev. Lett. 94, 106601 (2005).

[16] A. Matos-Abiague and J. Berakdar, Phys. Rev. B 70, 195338 (2004).

[17] A. Matos-Abiague and J. Berakdar, Phys. Rev. Lett. 94, 166801 (2005).

[18] B. Hillebrands, K. Ounadjela, Spin dynamics in confined magnetic structures, (Springer, Berlin, 2003).

[19] C. H. Back et al., Science 285, 864 (1999).

[20] Y. Acremann et al., Science 290, 492 (2000).

[21] I. Tudosa et al., Nature 428, 831 (2004).

[22] D. Atkinson et al., Nat. Mater. 2, 85 (2003).

[23] Th. Gerrits et al., et al., Nature 418, 509 (2002).

[24] D. Cole et al., Nat. Mater. 5, 305 (2006).

[25] F. Rossi and T. Kuhn, Rev. Mod. Phys. 74, 895 (2002).

[26] T. Chakraborty and P. Pietiläinen, Phys. Rev. B 50, 8460 (1994).

[27] N. E. Henriksen, Chem. Phys. Lett. 312, 196 (1999).

[28] D. Daems, S. Guerin, H. R. Jauslin, A. Keller, and O. Atabek, Phys. Rev. A 69, 033411 (2004).

[29] R. W. Robinett, Phys. Rep. 392, 1 (2004).

[30] A. Matos-Abiague and J. Berakdar, Europhys. Lett. 69, 277 (2005).

[31] Generalization to multi radial channels [30] can be handled in a similar way.

[32] Many-body effects on the ground-state distribution function are suppressed due to Pauli's principle. 